\title{
Shedding the light on spectrophotometry: the SpecUP educational spectrophotometer
}

Johan Nöthling, Patricia B. Forbes

Johan A. Nöthling, Patricia B. C. Forbes, "Shedding the light on spectrophotometry: the SpecUP educational spectrophotometer," Proc. SPIE 9289, 12th Education and Training in Optics and Photonics Conference, 92892B (17 July 2014); doi: 10.1117/12.2070728

SPIE Event: 12th Education and Training in Optics and Photonics Conference, 2013, Porto, Portugal 


\title{
Shedding the light on spectrophotometry: The SpecUP educational spectrophotometer
}

\author{
Johan A. Nöthling ${ }^{\text {a }}$, Patricia B.C. Forbes*a \\ ${ }^{a}$ Dept. of Chemistry, University of Pretoria, Lynnwood Road, Pretoria, 0002, South Africa
}

\begin{abstract}
Students often regard laboratory instruments as "black boxes" which generate results, without understanding their principles of operation. This is a concern, as the correct interpretation of analytical results and the limitations thereof is invariably based on an understanding of the mechanism of measurement. Moreover, a number of tertiary institutions in Africa have very limited resources and access to laboratory equipment, including that related to the field of photonics, which prevents students from getting hands-on practical experience. This paper addresses both of these challenges, by allowing students to assemble a low cost spectrophotometer, called the SpecUP, which is then used in a range of chemistry-related experiments. Students can vary instrumental parameters to observe the effects these changes have on their experimental results. The SpecUP costs less than 50 euro to build, as compared to $\sim 3000$ euro for commercial systems. Examples of the results obtained using the SpecUP in applied chemistry experiments are briefly presented here.
\end{abstract}

Keywords: photonics, spectrophotometer, educational, low cost, spectrophotometry

\section{INTRODUCTION}

Spectroscopy and the applications thereof are included in many undergraduate courses in the natural sciences, including chemistry, physics, biochemistry and food science. Students often do not get to fully understand the principles of spectrophotometry during practicals, however, as a consequence of limited instrument availability. Modern spectrophotometers are also designed in a manner that limits the handling of components, which unfortunately leads to students operating the instruments as "black boxes" into which samples are placed and which miraculously generate results.

A novel spectrophotometer was therefore designed, which would enable students to build their own instrument and then use it to generate analytical data in applied experiments, which include the optimization of the placement of the components of which it is made. The basic components of a spectrophotometer are shown in Figure 1. The concept was based on a "build your own" spectrophotometer of Tavener and Thomas-Oates ${ }^{1}$, which uses a light emitting diode (LED) with a prism in front of a slit as a light source, and a light-dependent resistor (LDR) as a detector. We required that less focus be placed on the construction of the electronic component of our instrument and most importantly it was mandatory that the components of the spectrophotometer be moveable to allow students to experience the consequences of changing their positioning on the results obtained. A novel design was thus developed and is called the SpecUP.

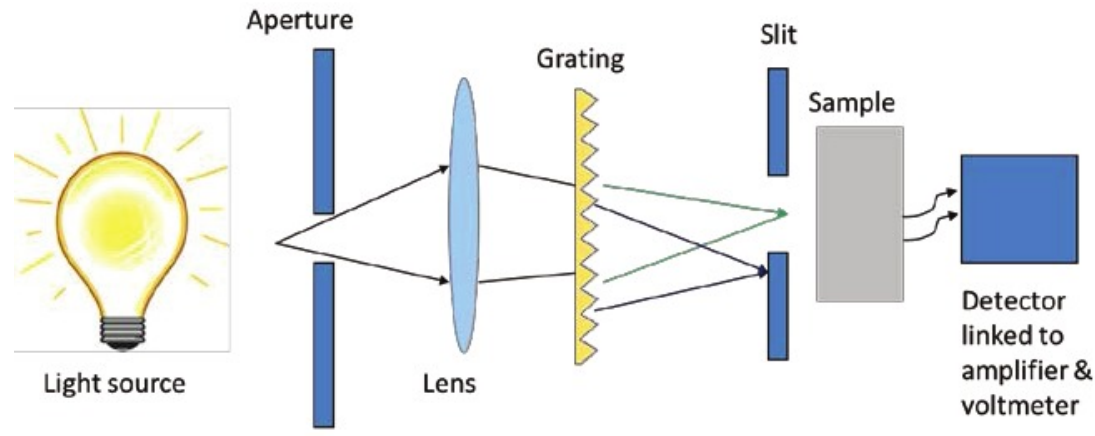

Figure 1. Basic components which can be employed in a simple spectrophotometer.

*patricia.forbes@up.ac.za; phone+27 (0)12 4205426

12th Education and Training in Optics and Photonics Conference, edited by

Manuel F. P. C. Martins Costa, Mourad Zghal, Proc. of SPIE Vol. 9289, 92892B

(C) 2014 SPIE, OSA, IEEE, ICO · doi: 10.1117/12.2070728

Proc. of SPIE Vol. 9289 92892B-1 


\section{METHODOLOGY}

\subsection{SpecUP design}

The SpecUP utilizes either a water clear white or coloured LED with a lens to focus the light as a source. When the white LED is used, a diffraction grating is employed as a monochromator. A LDR serves as a detector, as its resistance decreases with increasing light intensity. The components, including the sample cuvette, are mounted on retractable bars, which allow for their independent movement (Fig. 2). Two 9V batteries are used in conjunction with a simple printed electronic circuit ${ }^{1}$ of which the output voltage is measured using a low cost commercial multi-meter. A black cover is used to prevent stray light from interfering with the experiments. The cost of the components required for the SpecUP is less than 50 euro.

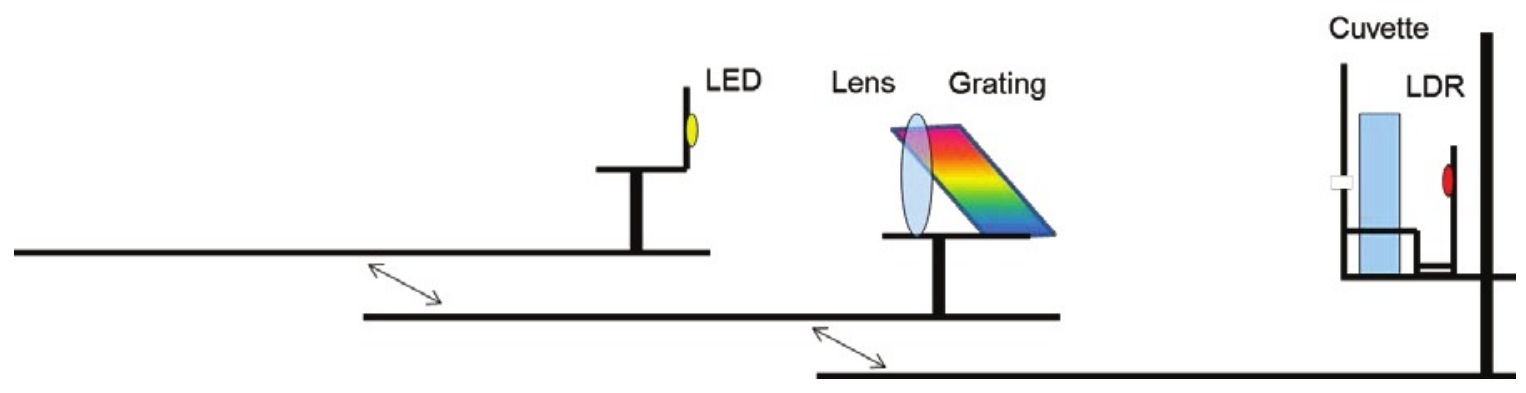

Figure 2. Side view showing the components of the SpecUP and their placement on retractable bars.

\subsection{Application 1: Beer-lambert Law}

In order to show the relationship between concentration and absorbance, as well as linear and non-linear regions in this regard, solutions of varying concentration of a coloured analyte are prepared and analysed. This can be done simply and cheaply (and without chemical disposal issues) by using commercial food colourants diluted in water. A coloured LED of complementary colour to that of the solution to be analysed is employed. This eliminates the need for a diffraction grating. The solutions of varying known concentrations are consecutively placed inside the sample cuvette and the voltage $\left(\mathrm{V}_{\text {sample }}\right)$ is recorded after the setup has been optimized in terms of component positioning and the solvent (water) and background voltage readings have been taken $\left(\mathrm{V}_{\text {solvent }}\right.$ and $\left.\mathrm{V}_{\text {dark }}\right)$. The voltage obtained for a sample of unknown concentration may also be recorded.

\subsection{Application 2: Generation of a spectrum}

A white LED is used in conjunction with a diffraction grating to produce the spectrum of a coloured compound in solution, such as potassium permanganate in water. The height of the sample cuvette is varied in a stepwise manner in order for different wavelength light to pass through the solution (Fig. 3). $\mathrm{V}_{\text {sample }}, \mathrm{V}_{\text {solvent }}$ and $\mathrm{V}_{\text {dark }}$ are recorded for each colour (sample position). A calibration curve can be constructed based on the wavelength of light reaching the sample for each sample position (described in terms of the number of turns of the wingnut holding the sample cuvette in place). 


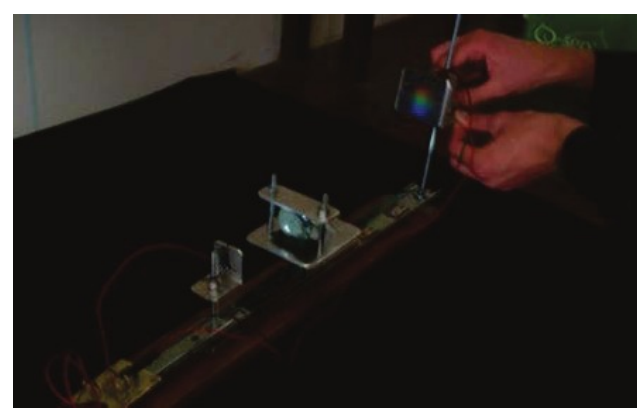

Figure 3. Changing the height of the sample cuvette in the SpecUP to vary the wavelength of light reaching the sample.

\section{RESULTS}

\subsection{Application 1: Beer-Lambert Law}

The results obtained for the solutions of known concentration are used to construct a calibration curve after each of the sample voltage readings have been converted to absorbance values $(A)$, where

$$
T=\frac{P_{\text {sample }}}{P_{\text {solvent }}}=\frac{V_{\text {sample }}-V_{\text {dark }}}{V_{\text {solvent }}-V_{\text {dark }}}
$$

and

$$
A=-\log T
$$

The concentration of the solution of unknown concentration can then be determined from the calibration regression line, an example of which is shown in Fig. 4. It is evident that excellent linearity is obtained even when sample dilutions have been roughly made (drops into $100 \mathrm{~mL}$ of water). Higher concentrations than those shown in Fig. 4 result in nonlinearity, as expected.

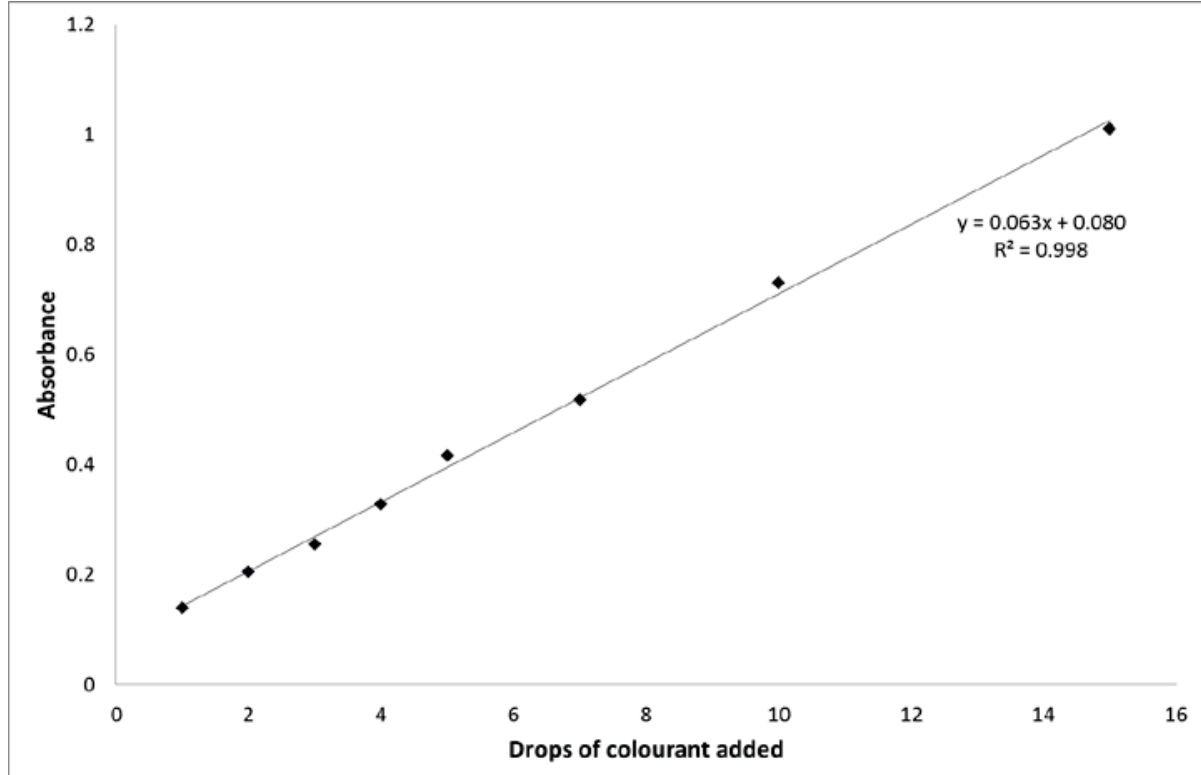

Figure 4. Calibration curve obtained with the SpecUP for blue food colourant dissolved in water. 


\subsection{Application 2: Generation of a spectrum}

The spectrum for potassium permanganate obtained with the SpecUP is comparable to that obtained with a commercial spectrophotometer (Fig. 5), although it lacks some of the fine spectral detail. The wavelength corresponding to the maximum absorbance for an analyte can be obtained in this manner, which can subsequently be used in additional experiments to determine unknown concentrations of solutions of this compound.

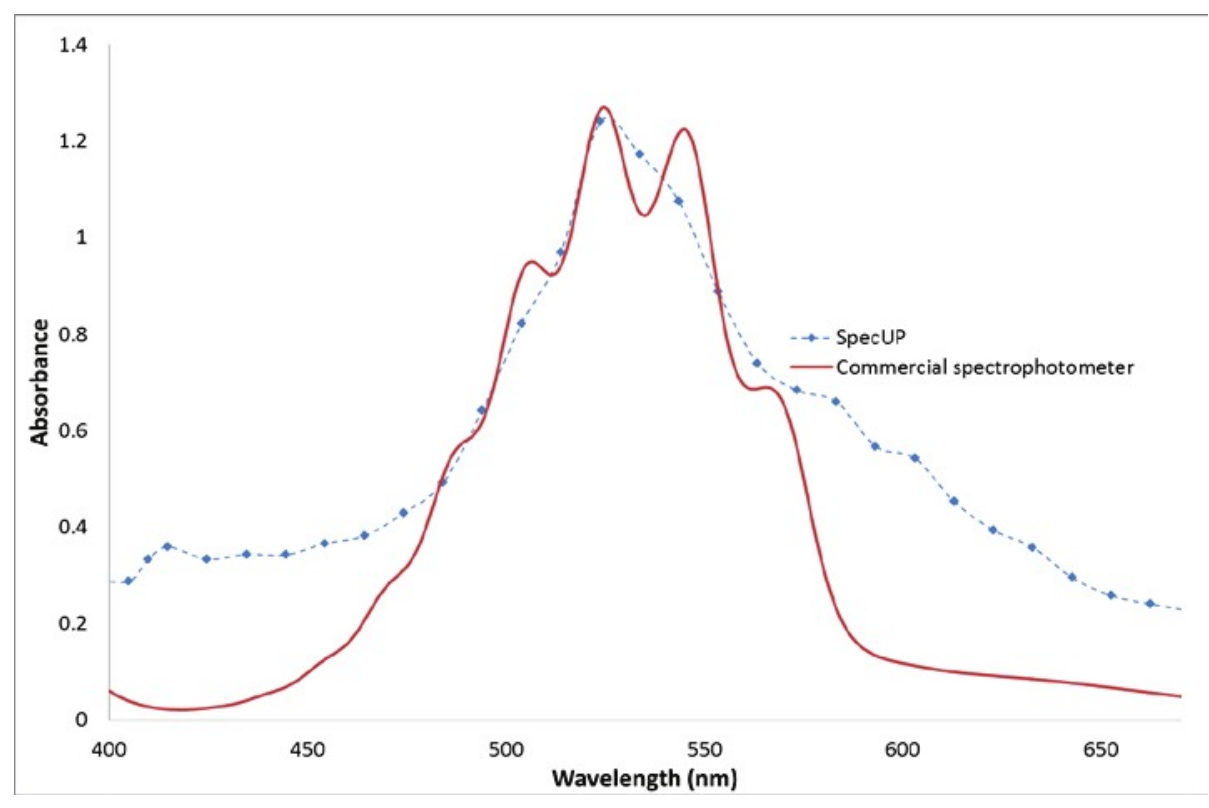

Figure 5. Comparison of the spectra of potassium permanganate obtained with the SpecUP and a commercial spectrophotometer.

\section{CONCLUSION}

The SpecUP spectrophotometer provides a cost effective and practical alternative to commercial instruments. It provides students with the opportunity to build their own instrument, which can then be used to generate analytically useful results. Additional application experiments in the field of chemistry include the determination of reaction kinetics where the development of colour occurs, such as in the iodine clock reaction, the determination of the alcohol content of wines as well as the colorimetric end point of titrations ${ }^{2}$. Experiments can also be designed for use in the teaching of a range of other undergraduate courses besides chemistry, such as in the fields of biochemistry and physics. Students should gain a much deeper understanding of spectrophotometry by the hands-on practical experience which the SpecUP provides.

\section{ACKNOWLEDGEMENTS}

The CSIR National Laser Centre (NLC) is thanked for project funding provided via the Photonics Initiative of South Africa (PISA) Human Capital Development Fund. Professor Andrew Forbes and Thomas du Plooy of the NLC are thanked for their technical assistance. Phakama Botha is acknowledged for her contributions to the development of the first prototype. Leon Engelbrecht of the University of Pretoria is thanked for buidling the electronics and Mrs Nöthling for manufacturing the SpecUP cover. Professor Thomas-Oates is noted for her interest and comments on the project.

\section{REFERENCES}

[1] Tavener, S.J. and Thomas-Oates, J.E., "Build your own spectrophotometer," Educ. Chem. 4, 151-154 (2007).

[2] Nazarenko, A.Y., "Optical sensors for manual and automatic titration in undergraduate laboratory," Spectrosc. Lett. 43, 555-560 (2010). 